\title{
Making handover better
}

\author{
Author: Emily McNicholas
}

\begin{abstract}
Aim
The handover process had been identified, by staff and the Care Quality Commission (CQC), as an area requiring improvement. As chief registrar, I was asked to identify problems with and to improve the on-call handover process within the medicine department.

\section{Methods}

An online survey was sent to all junior doctors participating in the on-call rota as well as nurse practitioners and coordinators from the hospital out of hours team. Questions were included about the timing, leadership, documentation, effectiveness and safety of the daily morning and evening handovers. The survey also asked respondents to suggest improvements to the handover process.
\end{abstract}

\section{Results}

The survey received 35 responses across different grades of staff. In general respondents were satisfied with evening handover, although only $37 \%$ felt it was standardised and consistent and $23 \%$ felt it was documented appropriately. Results for morning handover were less satisfactory. Only $41 \%$ felt that there was a formal handover meeting in the morning. Sixty-seven per cent had difficulties identifying the correct colleague to handover to and $56 \%$ felt that there was no standardisation.

In the free text responses, many respondents identified that there is no overlap between shifts in the morning and no embedded culture of a morning handover meeting. When asked for a preferred solution to this there was no overwhelming preference, but the largest percentage $(33 \%)$ wanted the base ward specialties to send a representative to take handover on behalf of their wards. This was implemented in April 2018 and the survey repeated in June 2018. This survey received 26 responses and showed some improvement in opinions regarding morning handover; now $96 \%$ agreed there was a formal meeting although $41 \%$ still had difficulties identifying the correct colleague. The survey also identified that increased use of the trust's information technology system could now permit a computerised handover; $92 \%$ agreed that using this would be a meaningful improvement. Handover will be developed further to incorporate a computerised handover starting with the new cohort of doctors in August.

\section{Conclusion}

The survey confirmed the generally held belief that the handover process needed improvement in order to bring it into line with the $R C P$ acute care toolkit 1 recommendations. It also helped to identify the most acceptable solutions to the problems presented by shift patterns, and guided continuous improvement within the handover process.

\section{Conflict of interest statement}

None declared. 